\title{
Memoria de Trabajo y Envejecimiento
}

\section{Working Memory and Aging}

\author{
Jorge Gontier B. ${ }^{1}$
}

\begin{abstract}
Resumen
Memoria de trabajo es un mecanismo cognitivo que almacena información temporalmente para ser usada y/o almacenada permanentemente. La memoria de trabajo puede ser afectada por el abuso de drogas y otras condiciones psicopatológicas. Existe también un importante efecto del envejecimiento en la rapidez y exactitud del procesamiento de información en la memoria de trabajo observado al comparar el desempeño de jóvenes y de adultos mayores en tareas de memoria de trabajo. El artículo es una revisión de investigaciones sobre el efecto de la edad en la memoria de trabajo. El creciente segmento de la población chilena mayor de 60 años hace necesario desarrollar conocimiento sobre su funcionamiento cognitivo para así facilitar su integración a la sociedad.
\end{abstract}

Palabras Claves: Memoria de trabajo, adulto mayor, efecto abanico.

\begin{abstract}
Working memory is a cogntive mecanism which stores information temporarily in order to use it and/or to store it permanentely. In addition, Working memory can be affected by drug abuse and other psychopathological conditions. Aging has also an important effect on the speed and accuracy of information processing in working memory. This effect has been observed when comparing young and older subjects studying working memory tasks. The purpose of this article is to describe the effect of aging in working memory. The fact that the chilean population of over 60 is continuously increasing requires the development of knowledge of their cognitive functioning to facilitate their social integration.
\end{abstract}

Keywords: working memory, aging, fan effect.

1 Programa de Master in Psychology. University of North Carolina at Wilmington. Estados Unidos. Unidad de Salud Mental. Hospital Penco-Lirquén. email: jig9450@uncw.edu 


\section{Memoria de Trabajo}

El concepto de memoria de trabajo aparece después de que los constructos de memoria a corto plazo y memoria a largo plazo fueran insuficientes para explicar algunos hallazgos científicos como por ejemplo el mecanismo por el cuál el material almacenado en la memoria a corto plazo pasa a formar parte de la memoria a largo plazo (Passig, 1994).

De acuerdo a Baddeley (2003), memoria de trabajo es un mecanismo cognitivo responsable por el almacenamiento temporal de información y su procesamiento. Baddeley propuso el concepto de memoria de trabajo después de observar durante un experimento a sujetos que tenían dificultad para ejecutar algunas tareas cognitivas mientras se les pedía retener secuencias de dígitos de número creciente.

Según Baddeley (1994), el concepto de memoria de trabajo abarca tres significados en psicología cognitiva: primero, como la producción de sistemas de modelos de arquitectura, esto es, memoria de trabajo es usada para referirse a un espacio dinámico en el que información específica y limitada es mantenida disponible para ser usada durante un particular período de tiempo. Un sistema de producción es una colección de reglas o parámetros que las personas usamos para retener y analizar información, establecer objetivos de lo que se pretende de esa información y verificar los resultados esperados de la información que se está procesando. Segundo, memoria de trabajo ha sido definida como un sistema que combina el almacenamiento y procesamiento de información. Usando esta última definición ha sido posible estudiar las diferencias que las personas presentan en un amplio rango de tareas cognitivas que implican la utilización de memoria de trabajo. Finalmente, la última y más aceptada conceptualización define memoria de trabajo como un sistema que se divide en tres componentes: lazo fonológico, esquema visuoespacial y ejecutivo central. Baddeley (2003) llamó al primer componen- te lazo fonológico. Esta división a su vez involucra dos subcomponentes: un sistema de almacenamiento temporal, que mantiene la información retenida por sólo pocos segundos. La información es almacenada brevemente y eventualmente se borraría sino es reforzada por el segundo subcomponente: este es un sistema de ensayo sub-vocal que no sólo mantiene información para evitar su olvido, sino también registra estimulación visual en la memoria permitiendo que los ítems almacenados puedan ser nombrados. El segundo componente llamado esquema espacial tiene la función de integrar información espacial, visual y posiblemente también kinestésica dentro de una representación unificada de manera de que la persona pueda operar con ella o almacenarla temporalmente. El último componente llamado ejecutivo central es el más complejo y difícil de entender. Este componente tiene la función de controlar a la memoria de trabajo como también coordinar los otros dos subsistemas: lazo fonológico y esquema espacial.

\section{Importancia de la Memoria de Trabajo en la Psicología}

Uno de los conceptos de más interés científico en psicología cognitiva en los últimos 20 años y que podría ampliar nuestro conocimiento a cerca de las diferencias cognitivo evolutivas en los adultos mayores es el concepto de memoria de trabajo. La base de datos PsycINFO de la American Psychological Association registra alrededor de 1500 artículos científicos que incluyen el concepto de memoria de trabajo en sus títulos desde 1990 hasta el año 2004 y más de 3500 lo citan en sus resúmenes. Más importante todavía, la Escala de Inteligencia Wechsler para Adultos en su tercera versión (WAIS III), ya traducida y que está siendo adaptada en nuestro País, incluye un índice de memoria de trabajo como factor de segundo orden al que contribuyen las subescalas Aritmética, Dígitos y Secuencias de Letras y Números (Satter and Ryan, 2001). Más aún, la memo- 
ria de trabajo ha sido una función cognitiva estudiada en diferentes ámbitos. En el campo relacionado con el abuso de drogas, se ha encontrado que el consumo de la droga ilegal MDMA (éxtasis) afecta negativamente el funcionamiento de la memoria de trabajo (Wareing, Fisk y Merphy, 2000); también lo hace el consumo crónico de alcohol (Ambrose, Bowden y Whelan, 2001) y por último la memoria de trabajo ha sido vinculada a la teoría cognitiva motivacional que explica la vulnerabilidad al alcoholismo crónico (Finn, 2002). La memoria de trabajo también se ha observado como una función interferida en pacientes con esquizofrenia (Manoach, 2003) en personas contagiadas por VIH (Basso y Bornstein, 2003) y en niños con problemas específicos de lenguaje (Montgomery, 2002). Más importante aún, la memoria de trabajo ha sido evaluada como una función altamente asociada a inteligencia (Chanquoy y Alamargot, 2002; Fry y Hale, 2000).

\section{La Memoria de Trabajo en el Desarrollo Individual}

El concepto de memoria a corto plazo ha sido insuficiente para explicar el efecto de la edad en tareas que requieren de memoria. Por otro lado, existe un gran número de estudios en el que la memoria de trabajo ha explicado con éxito las diferencias entre sujetos jóvenes y mayores de edad en el desempeño de tareas que implican memoria. Así también se han esclarecido mecanismos que están a la base del procesamiento cognitivo (Elousa y Lechuga, 1999).

De acuerdo al Instituto Nacional de Estadísticas de Chile, los adultos mayores son aquellas personas que tienen 60 o más años de edad (INE, 1999). En ese sentido, hoy en día la población adulta mayor de Chile ha crecido hasta casi igualar a la población joven y se estima que para el año 2020 el porcentaje de población mayor de 60 años será aproximadamente el mismo que la población menor de 15 años. Este cambio en la distri- bución de la población hace necesario que los profesionales psicólogos se interesen, conozcan e investiguen acerca de las características de ese grupo de edad de manera de brindar un servicio profesional de calidad y centrado en sus necesidades. En esta línea, el propósito de este artículo es revisar las principales investigaciones que vinculan memoria de trabajo con envejecimiento y en especial su funcionamiento en adultos mayores, describiendo los principales procedimientos y pruebas asociadas a su medición.

En relación al efecto de la edad en seres humanos, la memoria de trabajo ha sido estudiada desde una perspectiva evolutiva. En su estudio acerca de memoria de trabajo y control inhibitorio, Chiappe, Hasher y Siegel (2000) mostraron la existencia de importantes diferencias en el desempeño de sujetos de diferentes edades en una tarea que implicaba el uso de memoria de trabajo. En la investigación, los sujetos eran 665 individuos de edades comprendidas entre los 6 y 49 años. Durante la tarea, los sujetos tenían que leer y completar oraciones a las cuales les faltaba la palabra final. Luego de varias series de oraciones, los sujetos debían repetir todas las palabras que faltaban e inmediatamente continuaban con otra serie de oraciones. Como ha sido supuesto, la memoria de trabajo es la capacidad de almacenar y activar información mientras otras actividades son realizadas, entonces la memoria de trabajo fue medida de acuerdo al número de errores cometidos al tratar de recordar las palabras. Chiappe et. Al. (2000) explicaron los errores como una consecuencia de interferencia de información irrelevante (las palabras de los otros conjuntos de oraciones) que compitió con la nueva información que los sujetos tenían que recordar después de cada set de oraciones. La taza de errores disminuía entre los sujetos de edades entre los 6 y 20 años. Los individuos de edades entre los 10 y 20 años presentaron el mejor desempeño en la tarea. Por el contrario, los individuos de edad mayor de 20 años mostraban una 
significativa y gradual disminución de su desempeño. Después de los 30 años de edad, los sujetos tenían más dificultad para ignorar la información irrelevante y recordar la lista de palabras.

La velocidad con la que los contenidos son procesados exitosamente en tareas de memoria de trabajo es otra importante característica en la que es posible encontrar diferencias entre jóvenes y adultos mayores. Salthouse y Babcock (1991) midieron los diferentes componentes de memoria de trabajo intentando explicar la contribución de cada uno de ellos a las diferencias asociadas a la edad en el desempeño en tareas que evaluaban este concepto. Salthouse y Babcock definieron los tres componentes de memoria de trabajo como almacenamiento, que es la capacidad para recordar los estímulos; eficiencia en el procesamiento, como la capacidad de seleccionar correctamente la respuesta a un problema; y coordinación, o la habilidad para desempeñar dos actividades al mismo tiempo. Los tres componentes fueron medidos con tres tareas diferentes mientras que memoria de trabajo como todo un constructo fue evaluado con otros dos procedimientos. Los sujetos del estudio fueron 227 adultos de edades entre 20 y 87 años. El constructo de memoria de trabajo fue evaluado con una tarea de Extensión Computacional. En este procedimiento, los sujetos tenían que resolver problemas aritméticos (por ejemplo $3+$ 4) que eran presentados oralmente a un ritmo normal. Los participantes tenían que escoger la respuesta correcta entre tres alternativas. Después de un número específico de problemas aritméticos, a los individuos se les preguntaba por las respuestas correctas a los problemas antes resueltos. La otra tarea usada para medir memoria de trabajo como constructo fue Extensión de Listado. En esta tarea, los sujetos escuchaban una oración (por ejemplo: "el niño corrió con el perro") y ellos respondían a una pregunta oral ("¿Quién corrió?") eligiendo la alternativa correcta. Después de responder un número de proble- mas, a los sujetos se les pedía recordar y escribir las alternativas que ellos escogieron a cada problema. En relación a la evaluación de los componentes de la memoria de trabajo, las tareas llamadas Retención de Dígitos y Retención de Palabras, fueron utilizadas para evaluar el componente de capacidad de almacenamiento. En la tarea de Retención de Dígitos, los sujetos escuchaban una lista de dígitos para luego recordar y escribir dichos dígitos. La cantidad de dígitos era incrementada de tres hasta once dígitos, con una extensión de tres series. La tarea de Retención de Palabras consistió en el mismo procedimiento pero usando palabras como estímulos. El componente de eficiencia en el procesamiento fue medido a través de dos tipos de tareas, una aritmética y otra de comprensión de oraciones. La tarea aritmética consistía en resolver problemas aritméticos $(5+3=$ ?) presentados en una página y elegir la solución entre varias alternativas en no más de 20 segundos. La tarea de comprensión de oraciones tenía la misma estructura anterior pero con problemas de oraciones similares a la tarea de Extensión del Listado previamente explicada. Finalmente, una tarea de coordinación medía el componente de efectividad en la coordinación. Dos tipos de tareas de coordinación fueron administradas a los individuos en dos ocasiones separadas por 20 segundos. En una de ellas, algunos problemas aritméticos, similares a los ya explicados, eran presentados visualmente a los individuos y debían resolverlos mientras al mismo tiempo tenían que responder a una pregunta oral eligiendo entre tres alternativas. En la segunda tarea de coordinación, los problemas verbales eran presentados visualmente mientras problemas aritméticos eran presentados en la auditiva. Las medidas en cada tarea eran combinadas creando puntajes compuestos que reflejaban los diferentes constructos teóricos y señalaban el peso de cada constructo en explicar la varianza de las diferencias entre los participantes jóvenes y adultos mayores en las tareas de memoria de trabajo. 
Los resultados de este estudio mostraron que el incremento progresivo de la edad está asociado a una disminución del desempeño en todas las tareas que involucran memoria de trabajo. Por otro lado, el análisis de los componentes mostró que la eficiencia en el procesamiento era el componente que tenía más importancia para explicar la varianza en las diferencias de edad. Capacidad de almacenamiento y efectividad de coordinación explicaron parte de las diferencias de edad pero estos dos componentes a su vez fueron también influidos por eficiencia en el procesamiento. Por último, fue observada una importante relación entre memoria y velocidad de procesamiento, medida en el tiempo demorado en realizar cada ejercicio, pero el mecanismo que explica tal relación no ha sido aun especificado.

\section{Envejecimiento y Manejo de la Información Irrelevante en la Memoria de Trabajo}

Limitaciones importantes han sido encontradas en los procesos de codificación y recuperación de la información en memoria de trabajo en personas mayores de 60 años (Richardson, et al., 1996). En ese sentido es que ha sido propuesto que el proceso de codificación es interferido por material no relevante. Sujetos de más de 60 años de edad tienen mayores dificultades para suprimir la información irrelevante que compite con el material crítico que la persona está intentando codificar. En la etapa de recuperación de la información, las cogniciones no relevantes son reactivadas y los individuos de más edad tendrían gran dificultad para recordar el material relevante en la memoria.

Gerard, Zacks, Hasher y Radvansky (1991) observaron como una de estas limitaciones se encuentra particularmente presente en adultos mayores de 60 años de edad comparados con sujetos jóvenes de entre 18 y 20 años de edad cuando información irrelevante compite con la información objetivo debido a la dificultad para suprimir la activación de material irrelevante en la memoria de trabajo. Este déficit en el proceso de inhibición de información no relevante ha sido llamado efecto abanico. En el experimento de Gerard et al., los participantes fueron divididos en dos grupos: un grupo joven, constituido por estudiantes con edades comprendidas entre los 18 y los 20 años de edad y un grupo de edad mayor, constituido por sujetos con edades entre los 66 y los 72 años. La tarea involucraba una primera fase de adquisición. En ella, los participantes tenían que memorizar 18 frases que eran presentadas en la pantalla de un computador por siete segundos cada una. Las frases eran construidas con nueve diferentes tipos de profesiones y nueve diferentes tipos de actividades con la forma: "La persona (tipo de profesional) desempeñó una actividad". Tres diferentes niveles de efecto abanico fueron construidos en cada lista de oraciones que los sujetos tenían que memorizar. En el nivel mayor de efecto abanico, las oraciones involucraban tres tipos de hechos por cada persona y tres tipos de hechos por cada actividad. Así el exceso de información presente por cada parte de la oración constituía información posiblemente irrelevante que debería interferir en el proceso de codificación. En el nivel medio de efecto abanico, las oraciones involucraban dos hechos para cada profesión y dos hechos para cada actividad. En el nivel más bajo de efecto abanico, sólo un hecho era asociado para cada persona y actividad. Después de que la lista de oraciones era presentada, los participantes tenían que responder 18 preguntas a cerca de las oraciones y recibían retroalimentación negativa cada vez que erraban. La fase de adquisición terminaba cuando los sujetos respondían correctamente 18 preguntas. En el test de velocidad de reconocimiento, los sujetos tenían que decidir si una frase que era visualmente presentada era o no parte de la lista previamente aprendida. El número de oraciones fue 135 y eran divididas en tres bloques de 45 ítems. Consecuentemente, se 
esperaba que el grupo de adultos mayores tuviera más dificultad que el grupo más joven en reconocer las oraciones que habían aprendido especialmente en la condición en la que habían más hechos asociados con un particular profesional o actividad en las oraciones (mayor efecto abanico). Fueron registrados el tiempo de respuesta y el número de errores en el reconocimiento. Los resultados mostraron que el tiempo para aprender las oraciones, el tiempo de respuesta y la tasa de errores eran significativamente más altos en el grupo de sujetos de mayor edad comparado con los individuos jóvenes. Claramente fue observado que los sujetos de edades comprendidas entre los 66 y 72 años tenían más dificultad con la interferencia de información irrelevante al intentar recuperar información que los sujetos jóvenes de edades entre los 18 y 20 años. Gerard et al. explicaron estos hallazgos sugiriendo que los sujetos del grupo de mayor edad tenían deficientes mecanismos inhibitorios en el nivel atención. Esto permitió que más información irrelevante fuera activada y que una vez activada, ella permanecía por más largos períodos de tiempo. Esta información formaba asociaciones erróneas con conceptos similares y por lo tanto, los sujetos del grupo de edades entre 66 y 72 años tenían problemas inhibiendo la evocación de asociaciones irrelevantes en la tarea de adquisición. Luego en la tarea de reconocimiento, las asociaciones equivocadas producían interferencia causando una elevada tasa de falso reconocimiento.

En otro experimento relacionado, $\mathrm{Li}$ (1999), sugirió que el déficit en la selectividad de la información ocasionaba un desempeño deficiente en tareas de memoria de trabajo, especialmente cuando los ítems que son almacenados son muy similares. Para probar esta hipótesis, Li propuso que los sujetos mayores de edad deberían mejorar cuando la selección de la información es reducida. En sus dos experimentos los sujetos fueron adultos jóvenes de entre 19 y 24 años y adultos mayores de entre 65 y 75 años de edad.
En cada ítem de la tarea los sujetos tenían que resolver ecuaciones matemáticas con diferentes niveles de complejidad y se les pedía también memorizar el número final de la ecuación, un número extra o una palabra. Después de un set de ecuaciones, los participantes tenían que recordar los números de cada serie. La selectividad fue medida mediante la diferencia entre desempeño en la condición de no asociación (ecuación + memorizar una palabra) y la condición de asociación (ecuación + recordar un número). En el primer experimento los resultados mostraron que, comparados con el grupo más joven, los sujetos del grupo de más edad tenían más dificultad para recordar números asociados a las ecuaciones que para recordar palabras asociadas a las ecuaciones. Ellos concluyeron que, comparados con los jóvenes de 19 y 24 años de edad, en los adultos de entre 65 y 75 años de edad existe una incrementada dificultad para recordar material cuando hay semejanza entre la información que es procesada y la que es almacenada en la memoria.

La eficiencia inhibitoria para seleccionar información y evitar información irrelevante ha sido evaluada junto a otras variables tales como velocidad y memoria de trabajo como predictores de lenguaje en adultos de edad mayor a 55 años y jóvenes entre 17 y 26 años de edad (Kwong y Bouchard, 1995). Kwong y Bouchard estudiaron a sujetos jóvenes de entre 17 y 26 años comparándolos con adultos de mayor edad entre 55 y 78 años en su desempeño en tareas de memoria de trabajo y lenguaje. Memoria de trabajo fue medida con una tarea de Repetición Invertida de Letras en la que los participantes tenían que escuchar series de letras y después repetirlas pero en orden inverso, desde la última a la primera. Los ítems empezaban con dos letras e incrementaban en una en cada ejercicio. La eficiencia inhibitoria fue medida a través una tarea de Interferencia Color-Palabra en la que los sujetos tenían que leer palabras de colores (rojo, verde, azul, 
negro y amarillo) ignorando los colores incongruentes en los que cada palabra estaba escrita. La variable lenguaje fue medida en tres diferentes tareas de lápiz y papel. Comprensión de lectura fue evaluada por una tarea en la que los individuos leían seis historias para luego responder preguntas a cerca de ellas. Las preguntas eran hechas después de cada historia y las historias estaban visualmente disponibles mientras ellos respondían a las preguntas. La comprensión de oraciones fue evaluada por un test en el que los sujetos tenían que discriminar entre oraciones con sujetos pasivos y objetos pasivos. Los resultados de este estudio mostraron que los adultos de mayor edad muestran mayor interferencia al intentar inhibir información irrelevante cuando este material compite con otra tarea de procesamiento o conducta. Este efecto de interferencia fue llamado Stroop Interference Effect. Kwong y Bouchard encontraron que la velocidad de procesamiento y el efecto de interferencia son predictores importantes del desempeño de adultos de mayor edad en tareas de lenguaje. Consecuentemente, las personas con menos habilidad inhibitoria para suprimir estímulos irrelevantes en memoria de trabajo, tienen inferiores habilidades de procesamiento de lenguaje que las personas que poseen una mejor eficiencia en inhibir información irrelevante.

El factor inhibitorio ha sido observado como teniendo un importante efecto en la interferencia retroactiva en individuos de edad mayor (Hedden y Park, 2001). Hedden y Park definieron interferencia retroactiva como la situación en que la nueva información que ingresa a la memoria causa el olvido del material previamente aprendido. El otro tipo de interferencia estudiado por Hedden y Park (2001) fue la llamada interferencia proactiva. Ella opera por el proceso contrario: la información previamente aprendida que ha sido almacenada tempranamente en la memoria, compite con el material aprendido más tarde. En el experimento de Hedder y Park, los sujetos jóvenes tenían en promedio, 22 años. Los sujetos de más edad tenían un promedio de edad de 72 años y no se especifican rangos de edades para los dos grupos. En la tarea, los individuos tuvieron que memorizar una lista de 60 pares de palabras (pares A-B) que eran presentados durante cuatro segundos cada uno, en una pantalla de computador. Después de 60 presentaciones, los sujetos tenían un período de descanso de 16 segundos durante los cuales fueron instruidos a tratar de recordar las palabras previamente aprendidas. Luego, los participantes debían leer en voz alta tres veces tres diferentes pares de palabras. En un tipo de par, una de las palabras era la misma que había sido presentada en uno de los pares a aprender $(\mathrm{A})$ y la otra era una palabra diferente pero tenía una relación semántica con el par previamente aprendido (C). El otro par consistía en dos diferentes palabras no relacionadas con los pares anteriormente aprendidos (C-D). La tercera parte del experimento involucraba una tarea de reconocimiento en la que se presentaban 12 pares de palabras y los participantes tenían que identificar los pares que habían memorizado. Los pares ofrecidos para reconocimiento eran de tres tipos: pares que eran los mismos de la lista original (A-B); pares relacionados con la lista a memorizar (A-C); y pares no relacionados (C-D). Los resultados indicaron que aún cuando los individuos del grupo con el menor promedio de edad y los del grupo de mayor promedio de edad fueron interferidos retroactivamente, el grupo de sujetos con mayor promedio de edad mostró una desproporcionada interferencia retroactiva. Hedden y Park explicaron este efecto como una interferencia ocasionada por la lectura en voz alta de palabras que activaron contenidos en la memoria de trabajo. Durante la tarea de reconocimiento, los sujetos con mayor promedio de edad fueron ineficientes en borrar el material recientemente leído en voz alta y activado en la memoria de trabajo. Este efecto de interferencia ocurrió en la misma proporción para los pares relacionados y no relacionados semánticamente en la tarea de 
reconocimiento. Consecuentemente, la interferencia fue provocada por la activación de contenidos en la memoria de trabajo con una base vocal articulatoria más que semántica.

A pesar del hecho de que existe una importante evidencia para el efecto inhibitorio en la memoria de trabajo, Schelstraete y Hupet (2002) sugirieron que la resistencia a la información no relevante no es el efecto medido en la tarea de Interferencia. En su estudio, 151 adultos con edades entre 30 a 80 años fueron evaluados en dos tareas. Los sujetos fueron divididos en cinco grupos de edad: 30 sujetos con edades entre los 30 y los 39 años, 30 sujetos con edades entre los 40 y 49 años, 30 individuos de edades entre los 50 y 59 años, 31 sujetos con edades entre los 60 y 69 años y otros 30 sujetos con edades entre los 70 y 80 años de edad. La tarea llamada Extensión de Lectura fue usada para evaluar memoria de trabajo en información verbal. Los participantes debían leer en voz alta diferentes oraciones y al mismo tiempo memorizar la última palabra de cada oración. Sesenta oraciones fueron asignadas en tres grupos de veinte oraciones y cada grupo involucró cinco ejercicios empezando con dos oraciones e incrementando en una hasta alcanzar el número de 6 oraciones. Después de cada grupo de oraciones, los sujetos tenían que recordar y repetir la última palabra de cada oración. Dos tipos de medidas fueron registradas, el número total de palabras correctamente recordadas y el número de palabras incorrectamente recordadas de otras oraciones. En la tarea de interferencia, un procedimiento similar al usado en el estudio de Kwong y Bouchard fue utilizado: la tarea de Interferencia de color. Los resultados mostraron que la resistencia a la interferencia disminuía gradualmente con la edad en cada uno de los grupos. Las diferencias en la resistencia a la interferencia eran significativas entre el grupo de sujetos con edades entre los $30 \mathrm{y}$ 40 años y el grupo de sujetos con edades entre los 60 y 70 años. Sin embargo, Schelstraete y Hupet no encontraron ninguna relación significativa entre el número de palabras recordadas incorrectamente en la tarea de Extensión de Lectura y el puntaje en la tarea de memoria de trabajo. Fue sugerido que parte de las diferencias en memoria de trabajo entre los sujetos de diferentes grupos de edad eran explicadas por otra variable diferente del factor inhibitorio.

\section{Memoria de Trabajo en la Generación y Procesamiento de Imágenes}

El procesamiento de imágenes en la memoria de trabajo ha sido evaluado como diferente en personas de edad mayor y personas jóvenes. Bruyer y Scailquin (2000) estudiaron estas diferencias en 165 sujetos con edades entre 30 y 80 años. También dividieron a los sujetos en cinco grupos de igual número de integrantes por cada grupo de edad. Los grupos de edades eran los siguientes: el grupo más joven tenía entre 18 y 33 años, un grupo de edades entre los 34 y 46 años, un grupo con edades entre los 47 y 56 años, otro grupo con edades entre los 57 y 66 años y un último grupo con edades entre los 67 y 80 años. En la primera parte del experimento, los participantes tenían que dibujar la versión mayúscula de letras que eran previamente presentadas en su versión minúscula durante 500 milisegundos. Durante el entrenamiento, ocho ensayos fueron realizados involucrando las letras $\mathrm{L}$ y $\mathrm{O}$, otorgando retroalimentación positiva o negativa en cada respuesta. Inmediatamente después, los sujetos completaban 64 ítems experimentales sin retroalimentación y la dificultad de cada ejercicio era variada de acuerdo a la complejidad de cada letra. La segunda parte de este estudio involucró medidas de diferentes indicadores de eficiencia de memoria de trabajo. Para evaluar la función de almacenamiento de la memoria de trabajo, la tarea consistió en recordar y dibujar las 
últimas seis consonantes que eran presentadas en series de 6,8,10 o 12 consonantes. Ocho series de cada extensión eran presentadas al azar. Para la medición del almacenamiento en el componente de la memoria de trabajo llamado esquema espacial, los participantes tenían que observar y luego dibujar una matriz de celdas en la que el 50\% de las celdas eran previamente llenadas al azar. La tarea variaba la dificultad para recordar y para dibujar de acuerdo a la complejidad de cada patrón. La interferencia de la información no relevante en la memoria de trabajo fue evaluada con la tarea de interferencia previamente explicada. El componente de velocidad de procesamiento fue evaluado en sesenta ejercicios de dos pares de letras presentados simultáneamente, en los que los sujetos tenían que decidir rápidamente si las letras eran las mismas o eran diferentes.

Resultados de la primera parte del estudio mostraron que la edad de los participantes tenía una relación linear con el desempeño en la tarea. La exactitud de las respuestas disminuía con el incremento de la edad. En suma, la exactitud era más afectada por la complejidad del estímulo en el grupo de sujetos de edad entre 67 y 80 años que en los sujetos de edades entre los 18 y 33 años. Este último efecto fue importantemente significativo en individuos de 55 o más años de edad.

En la segunda parte del estudio el propósito era explorar las características de la memoria de trabajo que están relacionadas con las diferencias encontradas en la primera parte de la investigación. Bruyer y Scailquin (2000) encontraron que la dificultad en la generación y procesamiento de imágenes en la memoria de trabajo presente en sujetos de más edad, era reflejada en todas las medidas de los diferentes componentes. De esta manera, el efecto inhibitorio, la velocidad de procesamiento, el almacenamiento y la complejidad del estímulo fueron afectados por la edad en las tareas de memoria de trabajo.

\section{Memoria de Trabajo e Imitación de Gestos}

En un breve estudio, Dimeck, Roy y Hall (1998), trataron de relacionar desempeño en tareas de memoria de trabajo y el déficit que tienen las personas de edad mayor en imitación de gestos. En su estudio, Dimeck et al. (1998) propusieron que cuando un sujeto observa algunos movimientos de otra persona con propósitos imitativos, las representaciones de los movimientos observados deberían ser almacenados en la memoria de trabajo para después ser enviados al sistema motor y ejecutar luego el movimiento. En el experimento, el desempeño de adultos jóvenes y mayores de edad fue comparado en una tarea de imitación gestual concurrente y demorada. A los sujetos, 10 adultos mayores (edad promedio de 71 años) y 10 adultos jóvenes (promedio de edad de 25 años), se les pidió imitar nueve gestos manuales. Primero los sujetos reproducían los movimientos mientras el modelo estaba presente (concurrente) y luego reproducían los movimientos pero sin la presencia del modelo (demorada). Los gestos a reproducir no tenían un significado de importancia. La medida registrada fue el porcentaje de exactitud en tres independientes características de los gestos: orientación de la mano, posición de los dedos y ubicación. En todas esas variables, el desempeño de la tarea fue menos exacto en el grupo de sujetos con el mayor promedio de edad comparado con el grupo de sujetos con menor promedio de edad. Significativas diferencias fueron encontradas entre la reproducción concurrente y la demorada. La ejecución concurrente era mejor que la demorada. Más interesante, el grupo con mayor promedio de edad necesitaba de mayor tiempo para recordar el gesto en la imitación demorada. Los autores del estudio concluyeron que esta última diferencia encontrada se debía a la incrementada dificultad que tienen las personas de edad mayor de 70 años para mantener información en la memoria de trabajo. 


\section{Efectos de la Edad en el Funcionamiento Neural Relativo a la Memoria de Trabajo}

El efecto de la edad en la memoria operativa ha sido visto en la actividad cerebral de jóvenes y adultos. Golob y Starr (2000) estudiaron las diferencias de los componentes de potenciales evocados en la corteza cerebral durante una tarea de memoria de trabajo. Los sujetos eran 24 divididos en dos grupos de igual número: un grupo "joven" con edades de entre 18 y 22 años y un grupo "viejo" de edades entre los 60 y 79 años. La tarea consistió en memorizar una lista de 1, 3 ó 5 dígitos. Después de un período de tres minutos, los sujetos tenían que discriminar entre una serie de dígitos, cuáles estaban dentro de la lista previamente aprendida y cuáles no. Mientras los sujetos realizaban la tarea, la actividad eléctrica de su corteza cerebral era registrada con electrodos ubicados en áreas específicas del cráneo. Otras mediciones fueron tiempo de reacción y precisión expresadas en porcentaje de respuestas correctas. Golob y Starr encontraron que el grupo "viejo" edad tenía tiempos de reacción mayores que el grupo "joven". Esta diferencia se incrementaba en una relación lineal con la cantidad de dígitos. Mientras más dígitos eran presentados, mayor la lentitud en la respuesta de los sujetos de más edad. También esto ocurría en el grupo "joven" pero el incremento en la lentitud de respuesta era significativamente mayor en el caso del grupo "viejo". Se observaron también diferencias cualitativas entre estos dos grupos de edades diferentes en relación a la amplitud de la actividad eléctrica en el cerebro durante la tarea de memoria de trabajo. Cuando los sujetos del grupo "viejo" tenían que recordar la lista de números y responder si el número que era presentado era o no uno de los aprendidos anteriormente, la amplitud de la actividad eléctrica aumentaba significativamente en las áreas de la corteza auditiva. Más aún, este efecto era más importante cuando el ítem tenía más dígitos que recordar. El efecto opuesto fue observado en el grupo "joven".
Más evidencia acerca del efecto de la edad en la actividad cerebral relativa a memoria de trabajo ha sido mostrada usando mediciones de fluido regional de sangre en el cerebro (rCBR) (Esposito, Kirkby, Van Horn, Ellmore y Berman, 1999). El experimento de Esposito et al. (1999) consistió en comparar la activación de áreas cerebrales prefrontales durante dos tareas cognitivas en un grupo de 41 sujetos con un rango de edad de 18 a 80 años separados en grupos proporcionales por cada de 10 años de edad. Los sujetos fueron cuidadosamente seleccionados, no habían diferencias significativas en el nivel educacional y fueron descartados procesos patológicos con el uso de exámenes de resonancia nuclear magnética, tests de laboratorio, exámenes físicos, tests neuropsicológicos y entrevistas. Las tareas cognitivas involucraron la administración del Wisconsin Card Sorting Test (WCST) y del Raven's Progressive Matrices test (RPM) mientras el fluido de sangre en el cerebro era medido en la corteza prefrontal de los sujetos. El WCST ha sido significativamente asociado con memoria de trabajo e implica una tarea en la que el individuo tiene que aparear cartas con cuatro diferentes cartas modelos de acuerdo con tres atributos: color, forma y número. No se le dan claves al examinado, sólo retroalimentación después de cada respuesta. Una vez que el sujeto ha completado diez apareamientos correctos de acuerdo al primer atributo, el atributo es arbitrariamente cambiado pero sin informar de ello al examinado. Entonces, el examinado debe encontrar por sí mismo la nueva clave para realizar correctamente los siguientes apareamientos con las cartas modelos. El test termina cuando el sujeto ha completado diez asociaciones correctas consecutivas con seis diferentes atributos o cuando el sujeto ha asociado correcta o incorrectamente las 128 cartas. ElWCST requiere que el examinado genere representaciones internas a cerca de sus estrategias y resultados en sus previas asociaciones de cartas. Luego debe almacenar esta información para formular inmediatas y futuras respuestas o asociaciones con el estímulo modelo. El RPM 
test es considerado una medida de inteligencia general. En este test, los individuos tienen que observar una matriz de seis celdas con un patrón específico en el que hay una parte que falta. Los sujetos tenían que elegir entre diferentes alternativas, aquella que mejor calza con la parte que falta en el patrón. Después de la administración del WCST y el RPM, dos tareas controles sensoriomotrices y de asociación fueron llevadas a cabo. En general, un más bajo desempeño cognitivo en ambas tareas fue encontrado en los sujetos de mayores de 60 años de edad comparado con el desempeño de los sujetos de edades entre los 20 y 30 años de edad. Este desempeño disminuía gradualmente con la edad. Más interesantemente, Esposito et al. observaron que en la administración de WCST, algunas áreas que eran más activas en los sujetos de los grupos de edades entre los 20 y 40 años de edad, estaban desactivadas en los sujetos de edades entre los 60 y 80 años de edad en cuanto a los niveles de fluido sanguíneo cerebral. Las áreas de la corteza prefrontal dorsolateral izquierda DLPFC (BA 9/45-46) y del lóbulo parietal inferior (BA 39-40) fueron activadas durante el WCST y RPM en el grupo de sujetos de edades entre 20 y 40 años pero estaban significativamente menos activadas en los individuos mayores de 60. Este nivel de desactivación fue diferente que el registrado en el RPM. Otro importante efecto de la edad fue encontrado en la activación de áreas prefrontales del Hipocampus entre los sujetos de entre 20 y 30 años de edad y los de edad sobre 60 años en las dos tareas cognitivas. En el grupo de más de 60 años de edad, las áreas hipocampales se activaban durante la tarea de WCST pero estaban menos activadas durante el RPM. La inversa relación de activación fue encontrada en los sujetos de edades entre 20 y 30 años. Esposito et al. explicaron que el significativamente aumentado nivel de activación de las áreas hipocampales en los individuos mayores de 60 años durante la tarea de memoria de trabajo podría representar un mecanismo secundario de compensación al déficit de la significativa desactivación en las áreas prefrontales. Más aún, Espósito et al. sugirieron que este mecanismo compensatorio podría involucrar el uso de memoria a largo plazo para compensar el déficit de memoria de trabajo, dado que ambos tipos de memoria están funcionalmente relacionadas con las áreas prefrontal e hipocampal respectivamente.

\section{Memoria de Trabajo y Procesos Patológicos en Adultos Mayores}

También han sido encontradas diferencias en el desempeño de tareas de memoria de trabajo entre grupos de sujetos de edad avanzada sanos, jóvenes sanos y sujetos de edad mayor con demencia u otro proceso patológico cerebral (Cox, Stefanova, Johnsrude, Robbins y Owen, 2002). En su estudio, Cox et al. (2002) probaron el desarrollo de condicionamiento de conductas de elección. Los participantes enfermos eran 22 sujetos de ambos sexos con Parkinson de edades entre los 52 y los 64 años y 13 pacientes de ambos sexos con daño unilateral en la corteza prefrontal de edades entre 23 y 37 años. Los sujetos de los grupos control (uno para cada condición de enfermedad) eran sujetos sanos relativamente equivalentes en su número, edad y escolaridad a los pacientes de los grupos experimentales. La primera tarea fue llamada formación y consistió en adivinar, mediante una imagen de computador, en cuál de tres cajas estaba escondida una pelota roja. Si el sujeto lograba acertar, uno de tres patrones de estímulos era revelado y el sujeto tenía la posibilidad de recibir una recompensa (comida o estímulo melódico). Si por el contrario no acertaba, otro patrón de estímulos era revelado y había la posibilidad de recibir castigo en la forma de un sonido intenso. 180 Ejercicios fueron administrados en bloques de 20, 30, 40, 40, 30 y 20 respectivamente. Después de terminar cada bloque, los sujetos debían responder el número de veces que ellos habían adivinado la ubicación de la pelota roja. La segunda tarea era llamada juicio. En ésta, los participantes eran expuestos a pares de patrones y tenían 
que escoger uno. Había seis diferentes patrones, tres coincidían con los patrones de la tarea de formación y tres eran nuevos. Finalmente, en la etapa de preguntas, los sujetos tenían que responder porqué ellos habían preferido los tres patrones más elegidos de manera de excluir a aquellos sujetos que se habían dado cuenta del procedimiento de condicionamiento. Como era esperado, los sujetos del grupo control de edades entre los 23 y 37 años cometieron menos errores al responder cuantas veces habían encontrado la pelota roja que los sujetos del grupo control mayores de edad. De la misma manera, los sujetos sanos mayores de 50 años de edad se desempeñaron mejor en esta tarea que los pacientes con Parkinson y que los pacientes con daño en la corteza prefrontal. En ese sentido, los sujetos mayores de 50 años de edad del grupo control fallaron en mostrar preferencias condicionadas en la tarea de juicio. Más aún, los sujetos sanos y enfermos de ese mismo rango de edad preferían estímulos familiares independientemente del condicionamiento. Contradictoriamente, los sujetos sanos de edades entre los 23 y 37 años de edad y los con daño pre-frontal tendían a preferir estímulos nuevos. Pacientes con Parkinson y con daño cerebral no pudieron realizar las tareas de memoria de trabajo. Cox et al. propusieron que el déficit de memoria de trabajo en estos pacientes se debe a un procesamiento anormal de la información en el lóbulo prefrontal debido a un anormal funcionamiento del circuito de los ganglios basales.

\section{Conclusiones}

Memoria de trabajo como concepto multicomponente ha ayudado a explicar algunas características de los cambios cognitivos que sufren los individuos como efecto de la edad. Además, La memoria de trabajo ha sido definido y considerado a lo largo de la literatura revisada como un mecanismo complejo capaz de almacenar información, usar la información disponible, mantenerla por un periodo breve de tiempo o practicarla para almacenarla más permanentemente y coordinar acciones simultáneas que implican el uso de la información (Baddeley, 1994). Durante la vida de una persona, la memoria de trabajo sufre de un proceso de crecimiento que al perecer ocurre entre los 0 y 20 años y un proceso de deterioro normal como efecto del envejecimiento que comienza aproximadamente a los 30 años (Chiappe, 2000). Las diferencias más importantes son notadas en personas sobre los 60 años de edad y en aquellos que padecen de alguna patología degenerativa nerviosa o secuela de daño encefálico en la corteza prefrontal (Cox et al., 2003). El deterioro de la memoria de trabajo ha sido explicado por el llamado efecto abanico (Gerard et al., 1991). Este concepto involucra la dificultad de inhibir información irrelevante y formar asociaciones erróneas entre el material relevante y el irrelevante en el proceso de codificación. La recuperación de la información también sería afectada por estas asociaciones. A mayor cantidad de información no relevante y mayor su similitud con el material objetivo a la que las personas son expuestas, más dificultad habrá para los procesos de codificación y recuperación. Estos elementos complican significativamente más a los adultos mayores que a los jóvenes.

El correlato neurofisiológico de los procesos implicados en la memoria de trabajo parece estar vinculado a ciertas áreas de la corteza prefrontal. Comparados con los jóvenes, la menor eficiencia en el funcionamiento de la memoria de trabajo de los adultos mayores tendría también al parecer, un funcionamiento neurofisiológico diferente involucrando áreas que no son activadas en los jóvenes durante la ejecución y que implican específicamente el uso de memoria de trabajo (Esposito et al., 1999).

La literatura revisada dirige la evidencia hacia un efecto negativo de la edad en la memoria operativa, pero a pesar de que los trabajos definen y especifican claramente la conceptualización de memoria operativa, sus componentes y sus formas de medirla, no 
definen envejecimiento como variable y se dirigen simplemente a expresarlo como el efecto de la edad sin ser específicos acerca de los cambios en el desarrollo de la memoria operativa en función de las etapas del desarrollo cognitivo humano. Tampoco se hacen los alcances necesarios relativos a las diferencias específicas esperadas en los diferentes procesos degenerativos patológicos diferenciándolos de lo esperado normal para la edad. Por otro lado algunos trabajos revisados no especifican la distribución de los sujetos a los diferentes grupos de edad ni la dispersión de sus edades en cada grupo (Chiappe et al., 2000; Hedden y Park, 2001), mientras otros trabajos asignan sujetos a grupos con una amplitud en el rango de edad de más de 10 años (Cox et al., 2002; Golob y Starr, 2000; Bruyer y Scailquin, 2000; Kwong y Bouchard, 1995).

\section{Referencias Bibliográficas}

Ambrose, M., Bowden, S. \& Whelan, G. (2001). Working memory impairments in alcohol-dependent participants without clinical amnesia. Alcoholism: Clinical and experimental Research, 25, 185-191.

Baddeley, A. (2003). Working memory and language: an overview. Journal of Communication Disorders, 36, 198-208

Baddeley, A. \& Hitch, G. (1994). Developments in the concept of working memory. Neuropsychology, 8, 485-493.

Basso, M. \& Bornstein, R. (2003). Effects of past noninjection drug abuse upon executive function and working memory in HIV infection. Journal of Clinical and Experimental Neuropsychology, 25, 893-903.

Bruyer, R. \& Scailquin, J. (2000). Effects of aging on the generation of mental images. Experimental Aging Research, 26, 337-351.

Chanqouy, L. \& Alamargot, D. (2002). Working memory and writing: evolution of models and assessment of research. Annee Psychologique, 102, 363-398.
Chiape, P., Hasher, L. \& Siegel, L. (2000). Working memory, inhibitory control and reading disability. Memory and Cognition, 28, 8-17.

Cox, S., Stefanova, E., Johnsrude, I., Robbins, T. \& Owen, A. (2002). Preference formation and working memory in Parkinson's disease and normal ageing. Neuropsychologia, 40, 317-326

Dimeck, P., Roy, E. \& Hall, C. (1998). Aging and working memory in gesture imitation. Brain and Cognition, 37, 124-127.

Elousa, M. \& Lechuga, M. (1999). Diferencias relacionadas con la edad en el funcionamiento de la memoria operativa. Cognitiva, 11, 109-125.

Esposito, G., Kirkby, B., Van Horn, J., Elimore, T. \& Berman, K. (1999). Contextdependent, neural system-specific neurophysiological concomitants of ageing: mapping PET correlates during cognitive activation. Brain, 122, 963-979.

Finn, P. (2002). Motivation, working memory, and decision making: A cognitivemotivational theory of personality vulnerability to alcoholism. Behavioral and Cognitive Neuroscience Reviews, 1, 183-205.

Fry, A. \& Hale, S. (2000). Relationships among processing speed, working memory and fluid intelligence in children. Biological psychology, 54, 1-34.

Gerard, L., Zacks, R., Hasher, L. \& Radvansky, A. (1991). Age deficits in retrieval: the fan effect. Journal of gerontology, 46, 131-136.

Golob, E. \& Starr, A. (2000). Age-related qualitative differences in auditory cortical responses during short-term memory. Clinical Neurophysiology, 111, 2234-2244.

Hedden, T. \& Park, D. (2001). Aging and interference in verbal working memory. Psychology and aging, 666-681. 
Instituto Nacional de Estadísticas (1999). Chile y los adultos mayores: Impacto en la sociedad del 2000. Santiago. Santiago de Chile: Departamento de Servicios al usuario y difusión: Autor.

Kwong, S., \& Bouchard, E. (1995). Cognitive mediation of adult age differences in language performance. Psychology and Aging, 10, 458-468.

Li, K. (1999). Selection from working memory: on the relationship between processing and storage components. Agin, Neuropsychology and Cognition, 6, 99-116.

Manoach, D. (2003). Prefrontal cortex dysfunction during working memory performance in schizophrenia: reconcling discrpant findings. Schizophrenia research, 60, 285-298.

Montgomery, J. (2002). Understanding the language difficulties in children with specific languaje impairments: Does verbal working memory matter?. Amnerican journal of speech language pathology, 11,77-91.

Passig, C. (1994). Los sistemas de memoria. Revista de Psicología de la Universidad de Chile, 5, 27-34.
Reuter, P., Jonides, J., Smith, E., Hartley, A., Miller, A., Marshuetz, C. \& Koeppe, R. (2000). Age differences in the frontal lateralization of verbal and spatial working memory revealed by PET. Journal of Cognitive Neuroscience, 12, 174-187.

Richardson, T., Engle, R., Hasher, L., Logie, R., Stoltzfus, E. \& Zacks, R. (1996). Working memory and human cognition. New York, NY: Oxford University Press.

Salthouse, T. \& Babcock, R. (1991). Descomponing adult age differences in working memory. Developmental Psychology, 27, 763-776.

Sattler, M. (2001). Assessment of Children. Cognitive Applications. Fourth Edition. San Diego, CA: Jerome M. Sattler Publisher Inc.

Schelstraete, M. \& Hupet, M. (2002). Cognitive aging and inhibitory efficiency in the Deneman and Carperter's working memory task. Experimental Aging Research, 2002, 269-279.

Wareing, M., Fisk, J. \& Murphy, P. (2000). Working memory deficits in current and previous users of MSMA ("ecstasy"). British Journal of Psychology, 91, 181-188. 